\title{
Automatic spraying motion planning of a shotcrete manipulator
}

\author{
Gangfeng Liu ${ }^{1} \cdot$ Xianchao Sun $^{1} \cdot$ Yubin Liu ${ }^{1} \cdot$ Tao Liu $^{1} \cdot$ Changle Li $^{1} \cdot$ Xuehe Zhang ${ }^{1}$
}

Received: 3 June 2020 / Accepted: 5 January 2021

(c) The Author(s) 2021

\begin{abstract}
Shotcrete manipulator has been widely used in the construction of high-speed railways, coal mines and other tunnels. At present, workers are still using the remote controller to control the manipulator for spraying operations. There are problems such as high operation risk, large personal injuries, and low construction quality. In order to improve the automation of tunnel construction, this paper proposes a spraying motion planning method based on an 8R manipulator arm. On the basis of kinematics modeling and analysis of the shotcrete manipulator, method design and motion planning of shotcrete are carried out according to tunnel conditions, and verification experiments are carried out in actual tunnel. The experimental results show that the precision of the automatic spraying method is less than $45 \mathrm{~m}$, which can meet the requirements of actual construction.
\end{abstract}

Keywords Shotcrete manipulator $\cdot$ Manipulator automatic spraying $\cdot$ Redundant manipulator $\cdot$ Analytical method $\cdot$ Method of shotcrete operation $\cdot$ Motion planning $\cdot$ Block spraying

\section{Introduction}

In the 1960 s, on the basis of summarizing the tunnel construction experience of his country, Professor L. V. Rabcewicz of Austria proposed an advanced construction method "New Austrian Tunneling Method," which was based on the theory of tunnel engineering experience and rock mechanics. The foundation combines the anchor rod and shotcrete as a construction method as the main support method [1]. Shotcrete support is a key element of the "new Austrian Tunneling Method," which is the abbreviation of shotcrete support and bolt support. It is widely used in railway highway tunnels, underground buildings, various mines and water resources and hydropower engineering around the world, moreover promoted the rapid development of shotcrete technology.

Shotcrete is to use compressed air or other power to transport concrete mixed with a certain proportion along the pipeline to the nozzle, and the concrete is sprayed vertically onto the surface at a very high speed. The continuous impact of cement and aggregate during the spraying process relies on the quick-setting agent to press tightly in a short

Gangfeng Liu

liugangfeng@hit.edu.cn

1 State Key Laboratory of Robotics and System, Harbin Institute of Technology, Harbin, China time to form concrete with various strengths, thus forming a concrete supporting layer. At present, tunnel construction mainly adopts manual operation. Workers control the shotcrete manipulator through the handheld remote controller to perform spraying operations. There are many problems in this operation mode $[2,3]$.

(1) A large amount of dust generated during the spraying operation of the shotcrete manipulator will block the operator's line of sight, resulting in the operator not being able to accurately grasp the condition of the surface to be sprayed and the position and posture of the spraying nozzle, resulting in a low spray quality for manual operations.

(2) The situation in the tunnel is complicated and unstable, and there is a risk of rock burst and rockfall, which poses a threat to the personal safety of the workers.

(3) The rebound phenomenon during shotcrete will produce a large amount of dust. The dust contains a large amount of quick-setting agent. The dust inhaled by workers will cause great harm to the body and cause pneumoconiosis and even cancer.

(4) The operator generally needs to control multiple handles to manipulate multiple joints of the manipulator, which is difficult to operate and requires the operator to have excellent skills. At present, there is a shortage of skilled operators.

The best way to solve the above problems is to realize automatic spraying of the shotcrete manipulator. The automatic spraying of the shotcrete manipulator can not only 
free people from the harsh spraying environment, protect the safety and health of the workers, but also ensure the quality of the sprayed layer and improve the work efficiency. Therefore, it is very necessary to carry out research on automatic spraying of the shotcrete manipulator.

To realize the automatic spraying operation of the shotcrete manipulator. First, a 3-d laser scanner is used as an environmental sensor to complete the three-dimensional scanning of the surface to be sprayed and obtain point cloud data. Then, the point cloud data are processed to obtain the actual contour of the tunnel to be sprayed. By comparing the actual contour and the design contour, accurate location information of the various areas to be sprayed is obtained, therefore the square volume of sprayed concrete required can be calculated. Through the path planning of the shotcrete manipulator, the position and posture information of the spraying nozzle is obtained, and then the kinematics inverse solution is obtained to obtain the joint variables. Finally, the lower computer controls the movement of each joint to realize the automatic operation of the shotcrete manipulator.

\section{Related work}

Shotcrete manipulator is typical redundant manipulator. At present, there is little research on inverse kinematics of redundant manipulator. D.E. Whitney obtains the inverse kinematics solution of the redundant manipulator by minimum norm method [4]. Koker used neural network to calculate the inverse kinematics of redundant robots, and used genetic algorithm to optimize the accuracy of solution [5]. However, the above methods are difficult to meet the realtime requirements of industry, they are usually only used for offline calculations. S. Guo and H.J. Cheng proposed an algebraic method to solve the inverse kinematics of 7-DOF redundant manipulator by increasing the number of known variables. This method has strong real-time performance and small computation [6]. M. Shimizu, H. Kakuya and W. Yoon used the parametric method to study the SRS robot arm, but due to the limitation of the mechanical structure, this method is not suitable for our research object [7].

Intelligent controls can automatically drive intelligent machines to achieve control goals without human intervention. Applying intelligent control technology to the control of the shotcrete manipulator can solve the problem of complex and uncertain environment during shotcrete. Q. Zhang proposed an intelligent control theory of earth-rock dam compaction combining intelligent decision-making and automatic control to achieve intelligent control of the earth-rock dam compaction process and improve compaction quality and efficiency [8]. S. Ko, A. Nakazawa and Y. Kurose proposed a method of dynamic automatic control of motion to improve the accuracy of neurosurgical robots
[9]. W.B. Rowe, Y. Chen, J.L. Moruzzi and B. Mills applied intelligent control to the grinding process and developed a general intelligent grinding control system [10]. Z.X. Cai detailed the application of intelligent control in robot planning and control [11].

G Girmscheid and M.Y. Cheng have carried out related research work on the automatic spraying of the shotcrete manipulator. G. Girmscheid and S. Moser introduced a shotcrete machine with three working states (manual, semiautomatic, and fully automatic) under development. The shotcrete machine measures the actual contour of the tunnel through the laser measurement system and compares it with the designed contour of the tunnel. Contour comparison and calculation of the thickness to be sprayed, in order to plan the movement of the shotcrete manipulator, so as to realize the automatic operation of the wet spraying machine [12]. M.Y. Cheng improved a 6-R shotcrete manipulator, used a contour graph to measure the tunnel excavation surface, and used a simulation model to calculate the spray nozzle path, which improved the automation of the wet sprayer system [13]. R. Berenstein and Y. Edan proposed an automatic spraying technology that uses industrial cameras to capture the information of the target to be sprayed, and then uses the target detection method to set the automatic spraying task [14]. H. Wang analyzed the trajectory of the shotcrete manipulator, proposed an optimal trajectory planning method for shotcrete manipulator based on cubic spline interpolation, and completed the trajectory planning of the shotcrete manipulator using this method [15]. G.Z. Tan and Y.C. Wang proposed a time-optimal trajectory planning and trajectory control method for industrial robots, which can ensure the motion of a robot's hand along a specified path in Cartesian space has the minimum traveling time under the constraints on the boundary values of joint displacements, velocities, accelerations, and jerks [16]. S.F.P. Saramago and V.S. Junior proposed a method to compute the optimal motions of robot in the presence of moving obstacles. In this algorithm, the optimal traveling time and the minimum mechanical energy of the actuators are together to build a multicriterion function [17].

At present, the research on the automatic spraying of the shotcrete manipulator is mainly aimed at the tunnels with regular contour after excavation, but the tunnels are mainly excavated by blasting. There are two cases of over excavation and under excavation on the tunnel surface, so these studies have little practical application value. This paper presents an automatic spraying method of shotcrete manipulator based on the HPS3016C shotcrete machine of China Railway Construction Heavy Industry Corporation Limited, which combines three-dimensional environment recognition, robotics, robot motion planning and other technologies. The application of the automatic spraying method of the shotcrete manipulator can make the spraying manipulator adapt 
to the contours of tunnels of different sizes, accurately detect the over-under excavation of the surface to be sprayed, plan the spraying path, and automatically spray the concrete.

\section{Kinematic Analysis of Wet Spray Manipulator}

Accurate calculation of the position and posture of the shotcrete manipulator in space is a prerequisite for motion planning, so it is necessary to establish a mathematical model of the shotcrete manipulator and analyze its forward and inverse kinematics. The research object of this paper is HPS3016C, whose structure is shown in Fig. 1, is an 8R manipulator. The 8 degrees of freedom are: base rotation, first-arm pitch, first-arm extension, second-arm rotation, third-arm rotation, third-arm extension, nozzle horizontal rotation, and nozzle vertical rotation.

The D-H method is used to establish the mathematical model of the manipulator, and the link coordinate system of the robotic arm is established as shown in Fig. 2, and the corresponding D-H parameters are shown in Table 1.

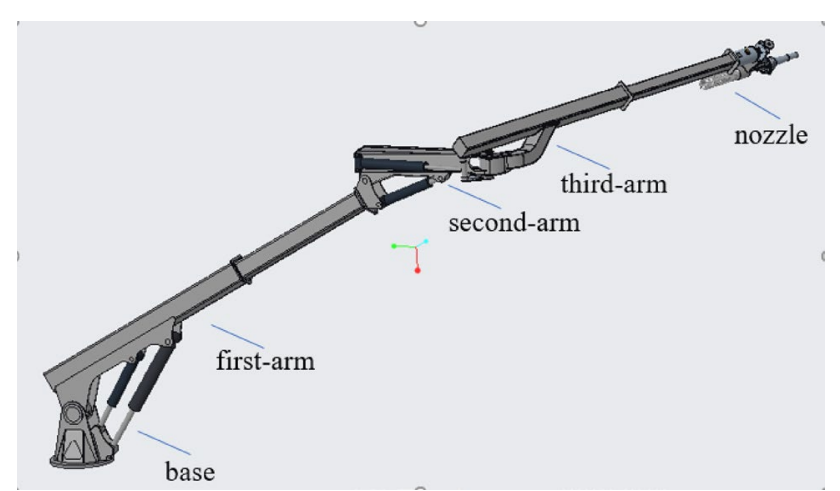

Figure. 1 HPS3016C shotcrete manipulator structure
Table 1 D-H parameters of shotcrete manipulator

\begin{tabular}{llllll}
\hline Joint number & Joint variable & $\theta$ & $d$ & $a$ & $\alpha$ \\
\hline 1 & $\theta_{1}$ & $\theta_{1}$ & $l_{1}$ & 0 & $90^{\circ}$ \\
2 & $\theta_{2}$ & $\theta_{2}+90^{\circ}$ & 0 & 0 & $90^{\circ}$ \\
3 & $d_{3}$ & 0 & $d_{3}+l_{2}$ & $l_{3}$ & $-90^{\circ}$ \\
4 & $\theta_{4}$ & $\theta_{4}+90^{\circ}$ & 0 & $l_{4}$ & $-90^{\circ}$ \\
5 & $\theta_{5}$ & $\theta_{5}+90^{\circ}$ & $l_{6}$ & $l_{5}$ & $-90^{\circ}$ \\
6 & $d_{6}$ & 0 & $d_{6}+l_{7}$ & 0 & 0 \\
7 & $\theta_{7}$ & $\theta_{7}$ & $l_{8}$ & 0 & $90^{\circ}$ \\
8 & $\theta_{8}$ & $\theta_{8}+90^{\circ}$ & $l_{9}$ & $l_{10}$ & 0 \\
\hline
\end{tabular}

The forward kinematics model based on the D-H parameter table can obtain the position and posture of the end of the robot arm at any joint angle or displacement. The results of positive kinematics of the shotcrete manipulator studied are as follows:

The vector form of the transformation matrix $T_{8}$ is given as follows:

$T_{8}=\left[\begin{array}{cccc}n & o & a & P \\ 0 & 0 & 0 & 1\end{array}\right]=\left[\begin{array}{cccc}n_{x} & o_{x} & a_{x} & P_{x} \\ n_{y} & o_{y} & a_{y} & P_{y} \\ n_{z} & o_{z} & a_{z} & P_{z} \\ 0 & 0 & 0 & 1\end{array}\right]$

$n_{x}=C_{8}$

$n_{y}=C_{7} S_{8}$

$n_{z}=S_{7} S_{8}$

$o_{x}=-S_{8}$

$o_{y}=C_{7} C_{8}$

$o_{z}=S_{7} C_{8}$

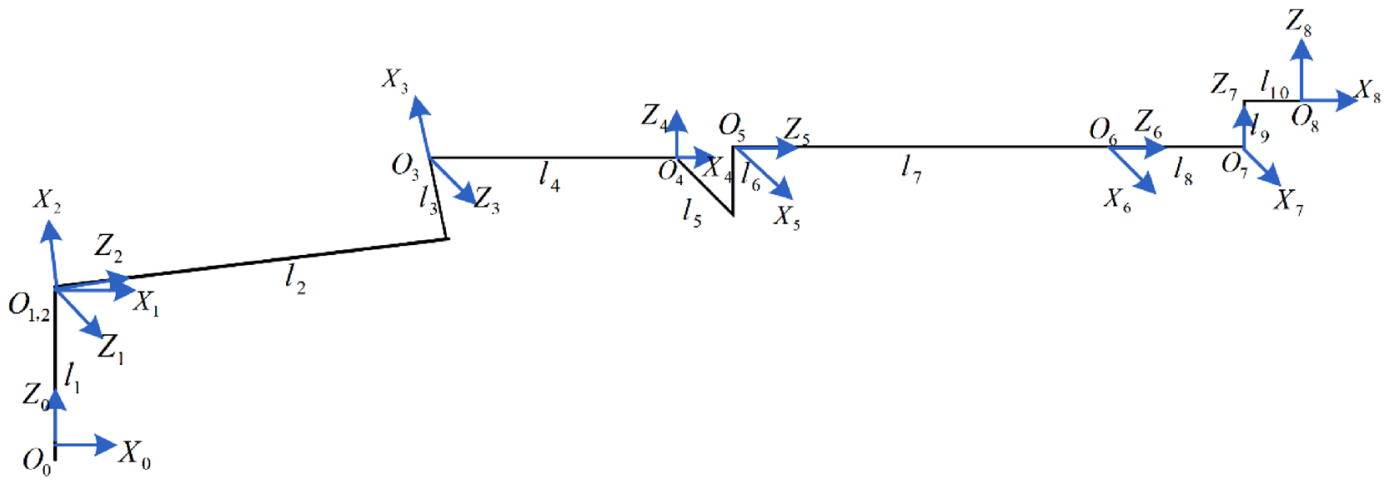

Figure. 2 HPS3016C shotcrete manipulator link coordinate system 


$$
a_{x}=0
$$

$$
a_{y}=-S_{7}
$$

$$
a_{z}=C_{7}
$$

$P_{x}=d_{6}+l_{7}+l_{8}+l_{4} C_{1}+l_{10} C_{8}-l_{3} C_{1} S_{2}+\left(d_{3}+l_{2}\right) C_{1} C_{2}$

$P_{y}=-l_{5}+l_{4} S_{1}-l_{9} S_{7}-l_{3} S_{1} S_{2}+l_{10} C_{7} S_{8}+\left(d_{3}+l_{2}\right) S_{1} C_{2}$

$P_{z}=l_{1}+l_{6}+l_{3} C_{2}+l_{9} C_{7}+l_{10} S_{7} S_{8}+\left(d_{3}+l_{2}\right) S_{2}$

The shotcrete manipulator studied has 8 degrees of freedom, so it belongs to redundant manipulator. There are infinite sets of solutions for the poses of specific points in the operating space. Considering the actual operation of the shotcrete manipulator, the third-arm must be consistent with the tunnel footage. We make joint 2 and 4 , joint 1 and 5 move together so that the sum of joint variables for joint 2 and 4 is 180 degrees and the sum of joint variables for joint 1 and 5 is 180 degrees. In this way, 2 degrees of freedom are reduced, and the problem studied becomes the inverse kinematics solution of the 6 degree of freedom manipulator. The specific inverse kinematics calculation process is given as follows:

(1) solve $\theta_{1}$.

The transformation matrix from the coordinate system 4 at the end of the third-arm to the base coordinate is given as follows:

$T_{4}=\left[\begin{array}{cccc}n_{4} & o_{4} & a_{4} & P_{4} \\ 0 & 0 & 0 & 1\end{array}\right]=\left[\begin{array}{cccc}n_{4 x} & o_{4 x} & a_{4 x} & P_{4 x} \\ n_{4 y} & o_{4 y} & a_{4 y} & P_{4 y} \\ n_{4 z} & o_{4 z} & a_{4 z} & P_{4 z} \\ 0 & 0 & 0 & 1\end{array}\right]$

Multiply the transformation matrix of the first 4 joints:

$T_{4}=\left[\begin{array}{cccc}-C_{1} C_{24} & -S_{1} & C_{1} S_{24} & -l_{3} C_{1} S_{2}-l_{4} C_{1} C_{24}+\left(d_{3}+l_{2}\right) C_{1} C_{2} \\ -S_{1} C_{24} & C_{1} & S_{1} S_{24} & -l_{3} S_{1} S_{2}-l_{4} S_{1} C_{24}+\left(d_{3}+l_{2}\right) S_{1} C_{2} \\ -S_{24} & 0 & -C_{24} & l_{1}+l_{3} C_{2}-l_{4} S_{24}+\left(d_{3}+l_{2}\right) S_{2} \\ 0 & 0 & 0 & 1\end{array}\right]$

From $(14)$ and $(15)$, the $(1,4)$ elements are equal, we can obtain:

$P_{4 x}=l_{4} C_{1}-l_{3} C_{1} S_{2}+\left(d_{3}+l_{2}\right) C_{1} C_{2}$

During the operation of the shotcrete manipulator, the end of the third-arm always moves in the same vertical section, so the coordinate of the shotcrete manipulator in the $\mathrm{X}$-axis direction in the basic coordinate system is a fixed value. Therefore, in Eq. (14), $\mathrm{P}_{4 \mathrm{X}}$ in the transformation matrix $\mathrm{T}_{4}$ is a constant. It is assumed that $\mathrm{P}_{4 \mathrm{X}}$ is a constant $\mathrm{m}$, that is:

$l_{4} C_{1}-l_{3} C_{1} S_{2}+\left(d_{3}+l_{2}\right) C_{1} C_{2}=m$

From Eqs. (6), (9), (12), and (17), we can obtain:

$\theta_{1}=\arctan \frac{P_{y}+l_{5}-a_{y} l_{9}-n_{y} l_{10}}{m}$

(2) solve $\theta_{2}$ and $d_{3}$.

From Eqs. (4), (10), (13), we can obtain:

$l_{3} C_{2}+\left(d_{3}+l_{2}\right) S_{2}=P_{z}-l_{1}-l_{6}-a_{z} l_{9}-n_{z} l_{10}$

Suppose: $P_{z}-l_{1}-l_{6}-a_{z} l_{9}-n_{z} l_{10}=J$.

From Eq. (17), we can obtain:

$-l_{3} S_{2}+\left(d_{3}+l_{2}\right) C_{2}=\frac{m}{C_{1}}-l_{4}$

Suppose: $\frac{m}{C_{1}}-l_{4}=K$.

From Eqs. (19), (20), we can obtain:

$d_{3}=\sqrt{J^{2}+K^{2}-l_{3}^{2}}-l_{2}$

$\theta_{2}=\arctan \frac{J\left(d_{3}+l_{2}\right)-K l_{3}}{K\left(d_{3}+l_{2}\right)+J l_{3}}$

(3) solve $\theta_{4}$.

Since the sum of the joint variable of joint 2 and the joint variable of joint 4 is $180^{\circ}$, the joint variable $\theta_{4}$ is given as follows:

$\theta_{4}=180^{\circ}-\theta_{2}$

(4) solve $\theta_{5}$.

Since the sum of the joint variable of joint 1 and the joint variable of joint 5 is $180^{\circ}$, the joint variable $\theta_{5}$ is given as follows:

$\theta_{5}=180^{\circ}-\theta_{1}$

(5) solve $d_{6}$.

Substituting Eq. (1) and Eq. (17) into Eq. (11) gives:

$P_{x}=d_{6}+l_{7}+l_{8}+n_{x} l_{10}+m$

so:

$d_{6}=P_{x}-l_{7}-l_{8}-n_{x} l_{10}-m$

(6) solve $\theta_{7}$.

From formula (9), we can obtain:

$\theta_{7}=-\arcsin a_{y}$

(7) solve $\theta_{8}$. 
From formula (12), we can obtain:

$\theta_{8}=\arccos n_{x}$

When the target position and posture of the nozzle are known, the inverse kinematics can be used to accurately calculate the joint value. The motion planning of the shotcrete manipulator is actually planning the target position and posture of the manipulator nozzle in the tunnel space. The target position and posture of the nozzle are determined by path planning.

\section{Operation planning of the shotcrete manipulator}

\subsection{Automatic spraying operation method of the shotcrete manipulator}

In order to improve the quality of concrete spraying, reduce the amount of rebound of concrete, and improve the compressive strength of concrete, it is necessary to investigate the characteristics of the shotcrete operation, explore the influence of spraying parameters on the quality of concrete, and design a reasonable operation process and spraying parameters. For the automatic spraying operation of the shotcrete manipulator, we studied the automatic spraying method of the shotcrete manipulator.

The automatic spraying operation method of the shotcrete manipulator adopts spraying in stages and zoning strategies. The flow of the automatic spraying operation is shown in Fig. 3. The spraying operation is divided into four stages in a staged spraying mode. These four stages are: Patching, the First padding, the Second padding, and Sweeping. The purpose of Patching is to fill up the more obvious depressions on the surface to be sprayed after blasting, which is beneficial to the subsequent spraying step; First padding is to fill the concrete between the wall surface to be sprayed and the back of the steel arch; Second padding is to fill the concrete between the steel arch; Sweeping is to cover the surface layer of the steel arch frame with concrete of $2 \mathrm{~cm}$ thickness. In the Second padding, a zoned injection strategy is adopted. As shown in Fig. 4, the tunnel to be sprayed is divided into 7 areas, each of which is about $2 \mathrm{~m}$ high. The areas are numbered from left to right and from bottom to top. The advantage of this zoned injection method is that the steel arch can be evenly stressed. When spraying, the steel arch is arranged along the tunnel outline, and the accumulation of concrete on one side will cause excessive stress on one side of the steel arch, which will cause deformation of the steel arch. This partitioning method can avoid uneven stress of the steel arch and the spray sequence from bottom to top can play the role of fixing the steel arch first.
In the shotcrete process, when the mixing ratio of raw materials such as cement, gravel and accelerator is determined, it is necessary to select reasonable spraying parameters to improve the quality of the concrete. The injection parameters mainly include spraying distance, spraying angle, and the thickness of a single spraying.

The method of shotcrete is used to support the roadway, that is, under the action of compressed air, the concrete is sprayed to the rock surface at a higher speed. The concrete and the rock surface collide and bond first, then continuously accumulate and tamp, then undergo condensation hardening, and finally form a solid concrete supporting layer. In the above series of links, selecting the appropriate injection distance is conducive to the realization of each link. If the distance between the spray gun and the surface to be sprayed is close under a certain spraying speed, when the mixture collides with the rock surface, the coarse aggregate particles in the concrete cannot bond well with the mortar base, rebound more, and the rebound rate will increase. In addition, under the action of wind pressure, the particles in the concrete have fast speed and large kinetic energy, which may wash away the concrete already bonded on the surface to be sprayed. If the distance between the spray gun and the surface to be sprayed is far away, the concrete blown to the rock surface will lose a large amount of kinetic energy under a certain wind pressure, which will not only reduce the bonding amount of fine aggregate, deteriorate the compactness of coarse aggregate, but also affect the compressive strength. When the distance between the spray gun and the surface to be sprayed is far away, the spraying speed direction will deviate from the horizontal direction due to the self-gravity of the mixture. The spraying speed can be divided into vertical direction and horizontal direction, in which the speed in the horizontal direction is smaller and the speed in the vertical direction is larger. At the same time, under the condition of long spraying distance, the impact force on the surface to be sprayed is reduced, and the compactness of the concrete layer is also reduced. Considering comprehensively, the spraying distance of concrete is generally selected between $1.2 \sim 2 \mathrm{~m}$. In the Patching stage, considering the small range to be sprayed during pit filling, the spraying distance is set at $1.2 \mathrm{~m}$. During the First padding and the Second padding, the injection distance is set at $1.5 \mathrm{~m}$. When Sweeping, considering that the far spraying distance can improve the uniformity of spraying, the spraying distance is set at $2 \mathrm{~m}$.

During shotcreting operation, for a relatively flat surface to be sprayed, when the nozzle is aimed at the rock surface for vertical spraying, that is to say the spraying angle is 90 degrees, the rebound amount of concrete will be relatively small. For the surface to be sprayed that is uneven or covered by steel mesh, when the angle between the nozzle and the rock surface is less than 70 degrees, the impact force of the larger coarse aggregate particles in the concrete after 
Figure. 3 Flowchart of automatic spraying operation of the shotcrete manipulator

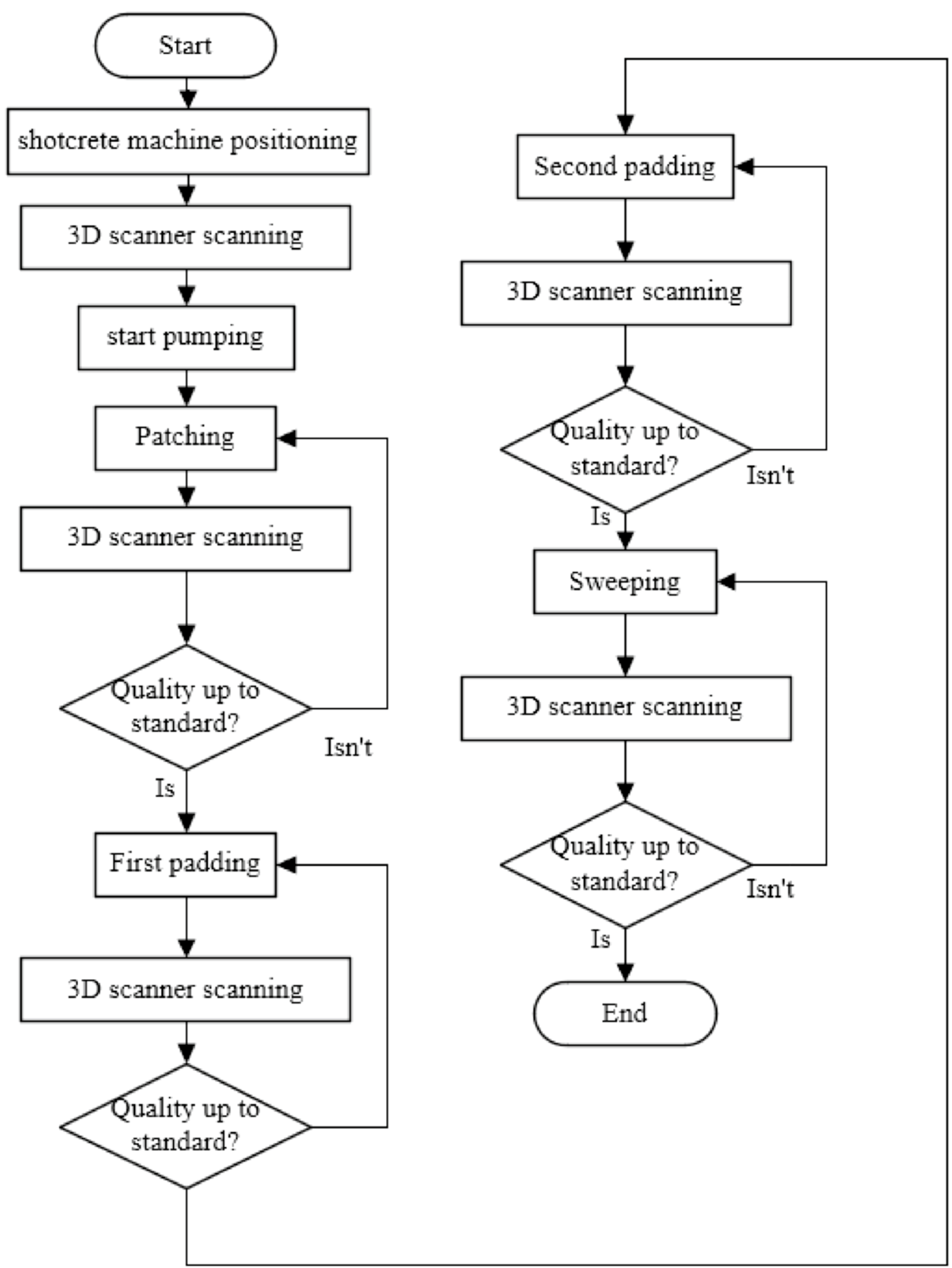

colliding with the rock surface is small, the concrete particles are not suitable for bonding, the rebound amount is large, and the compressive strength will also decrease, so the angle between the spray gun and the rock surface in this case should not be less than 70 degrees. When shotcrete manipulator is used for operation, the spraying angle is mainly determined by the initial posture of the nozzle and the rotation amount of the 7 th rotating joint. During the whole spraying operation, the initial posture of the nozzle is kept perpendicular to the wall surface to be sprayed, but the rotation amplitude of the 7 th joint is different in each stage. In the Patching stage, in order to prevent concrete from splashing to the rock surface around the pit, the 7 th joint is fixed, that is to say the rotation amplitude of the 7th joint is 0 . During the First padding and the Second padding, the rotation amplitude of the 7 th joint is set at $0 \sim 15$ degrees. When Sweeping, the rotation amplitude of the 7 th joint is set at $0 \sim 20$ degrees due to the increase of the spraying distance.

After the concrete enters the nozzle, the fine aggregate particles such as mortar will bond with the exposed rock surface under the action of compressed air to form a mortar base. However, if the nozzle stays in this position for a long time, the concrete bonded to the mortar base will increase. Due to the gravity of the concrete itself, the concrete may fall if the cohesive force is less than the gravity. Especially when the nozzle is aligned with the arch and the 


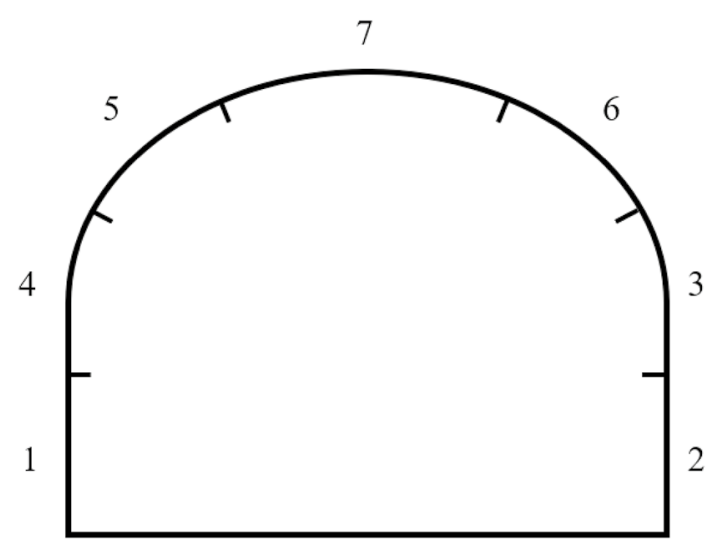

Figure. 4 Schematic diagram of tunnel zoning

angle between the nozzle and the surface to be sprayed is large, if the initial concrete spraying thickness is too large, the concrete rebound rate will increase. If the spray gun is aimed at the rock surface for a short period of time, the fine aggregate particles in the concrete can be well bonded on the rock surface to form a relatively uniform mortar base layer, but the thickness of the mortar base layer is relatively thin. If this rock surface is sprayed again, there will be less adhesion between the large-particle aggregate in the concrete and the mortar base, which will not only increase the rebound but also reduce the compressive strength of the concrete layer. Therefore, the thickness of a single spraying should not be too large or too small. However, for the automatic spraying of the shotcrete manipulator, the spraying times can be increased by adopting a smaller single spraying thickness, and the spraying uniformity can be improved to a great extent by combining the mode of selecting mutually interpenetrated spraying points in adjacent spraying. In the three stages of Patching, the First padding and the Second padding, the single thickness is $30 \mathrm{~cm}$.

In this way, the operation flow and spraying parameters of the automatic spraying operation of the shotcrete manipulator are all determined.

\subsection{Motion planning of the shotcrete manipulator}

In a complex and changeable tunnel space, according to the tunnel feature information obtained by 3D reconstruction, the trajectory planning of spraying is carried out. Referring to the experience of manual spraying, this paper proposes a block spraying strategy and uses this to carry out motion planning for the spraying manipulator.

The 3D reconstruction point cloud data are divided into blocks, and the area to be sprayed is divided into multiple rectangular unit blocks, as shown in Fig. 5. The center point of each unit block is taken as the spray point. During operation, the spray nozzle reaches each spray point in turn,

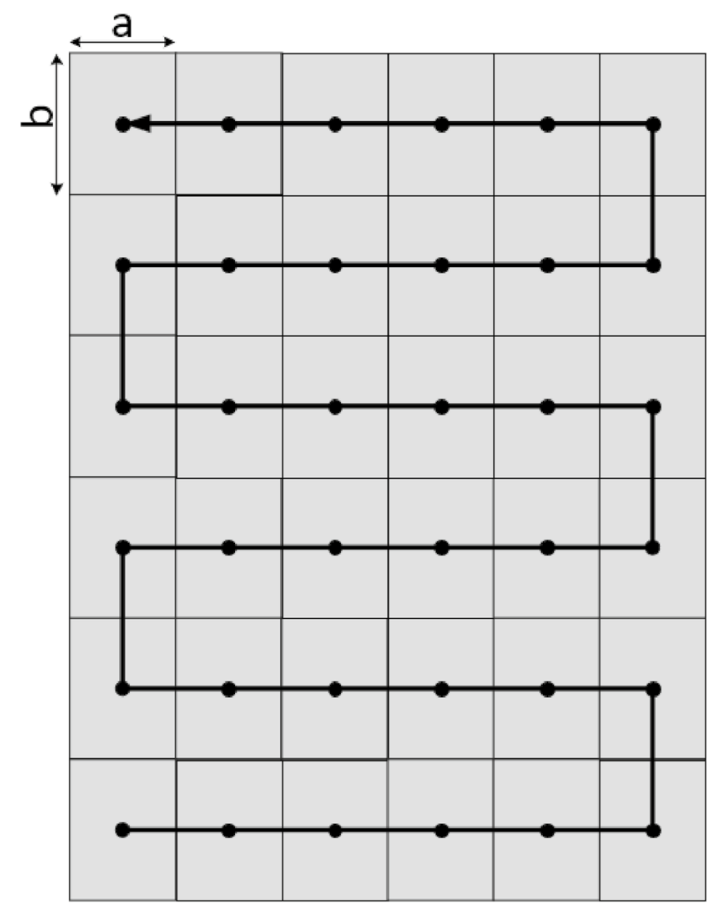

Figure. 5 "Z" shaped spraying path

and the horizontal and vertical rotation of the spray nozzle makes the concrete evenly cover the small block area. The spray points are connected by a "Z" shaped path. To make the spray nozzle follow this " $Z$ " shape path in the working space, the third-arm of the shotcrete manipulator can be kept parallel to the ground and the tunnel footage, and then use the expansion and contraction degrees of freedom of the third-arm. This is the constraint for solving the inverse kinematics of the HPS3016C shotcrete manipulator. A "Z" shaped path is used, making full use of the configuration characteristics of the HPS3016C shotcrete manipulator, realizing that the manipulator arm uses only one degree of translational freedom during most movements, which greatly simplifies motion control.

At each spraying point, the spray nozzle performs continuous reciprocating horizontal rotation and intermittent vertical rotation, so that the sprayed concrete can evenly cover each unit block. The length "b" and width "a" of the unit block are calculated from the angle range of the horizontal rotation and vertical rotation of the spray nozzle and spray distance, which is used as the basis of the block division. By processing the point cloud data after block division, the required spraying volume of each unit block is obtained, thereby determining the residence time of the spray nozzle at each spraying point.

In this way, the trajectory of the spraying robot arm can be completely determined. The trajectory of the spraying robot arm is divided into two parts. On the one hand, 
the movement path of the spray head between each spraying point forms a "Z" shaped path, on the other hand, the horizontal and vertical rotation of the spray nozzle at the spraying point.

The movement of the nozzle at the spraying point is a simple joint rotation. This paper mainly studies the trajectory planning of the nozzle moving in a " $\mathrm{Z}$ " shaped spraying path.

When spraying, according to the determined "bow" trajectory, the trajectory of the spray gun at the end of the shotcrete manipulator is usually straight and the posture does not need to be changed, as shown in Fig. 6 .

The pose transformation of the joint points $\mathrm{A}$ and $\mathrm{B}$ at the end of the mechanical arm can be considered as the translation of the tool coordinate along $\vec{\lambda}$. If the transformation matrix is ${ }_{A}^{B} T$, then there are:

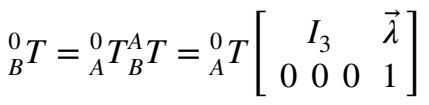

$I_{3}$ is a third-order identity matrix, $\vec{\lambda}=\left({ }_{A}^{0} R\right)^{T}\left({ }_{B}^{\overrightarrow{0} P}-\overrightarrow{{ }_{A} P}\right)(30)$.

If the linear motion process of the mechanical arm is acceleration, uniform speed and deceleration, and the whole linear motion time is $\mathrm{T}$ and the acceleration and deceleration time is $\Delta T$, then the motion speed curve at the end of the mechanical arm is trapezoidal, as shown in Fig. 7.

The position of the terminal of the manipulator during the movement is given as follows:

${ }_{B}^{0} T={ }_{A}^{0} T T(t)$

among them:

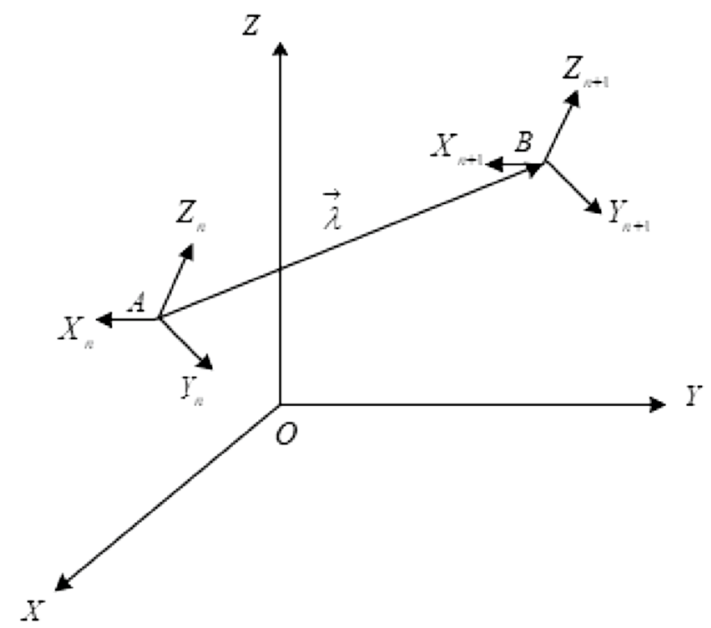

Figure. 6 Linear trajectory motion coordinate system

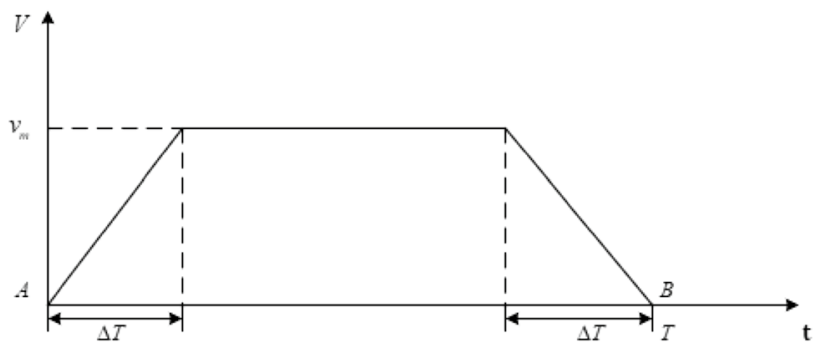

Figure. 7 Speed graph of the terminal of the manipulator

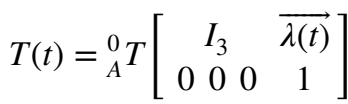

Assuming that $\overrightarrow{\lambda(t)}=\frac{\vec{\lambda}}{|\vec{\lambda}|} f(t)$, thus:

$f(t)=\left\{\begin{array}{cc}\frac{v_{m} t^{2}}{2 \Delta \Delta} & 0 \leq t \leq \Delta T \\ \frac{v_{m}(2 t-\Delta T)}{2} & \Delta T \leq t \leq T-\Delta T \\ v_{m}(T-\Delta T)-\frac{v_{m}(T-t)^{2}}{2 \Delta T} & T-\Delta T \leq t \leq T\end{array}\right.$

The pose matrix of the end of the shotcrete manipulator at any time in the process from point $\mathrm{A}$ to point $\mathrm{B}$ can be obtained from Eq. (31), and the obtained pose matrix can be used to obtain the angular position and rotation speed of each joint through the inverse kinematics of the mechanical arm and the Jacobi matrix.

In order to verify the rationality of this path planning method, as shown in Fig. 8, the shotcrete simulation system of the shotcrete manipulator was built using VREP softness. Let the nozzle take a "bow" shaped path to get the speed curve of each joint as shown in Fig. 9. It can be seen from the figure that the horizontal movement of the spray nozzle along the "Z" shape is mainly achieved by the sixth straight joint, and the longitudinal movement of the spray nozzle along the " $Z$ " shape is mainly achieved by the second,

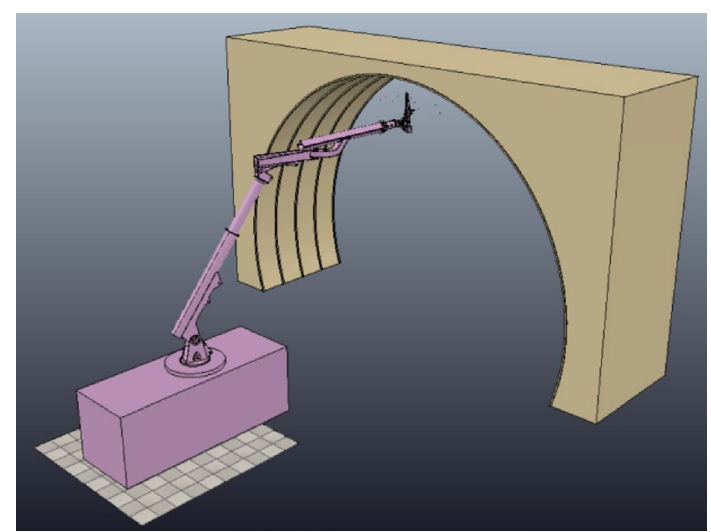

Figure. 8 Wet spraying robot arm spraying simulation system 


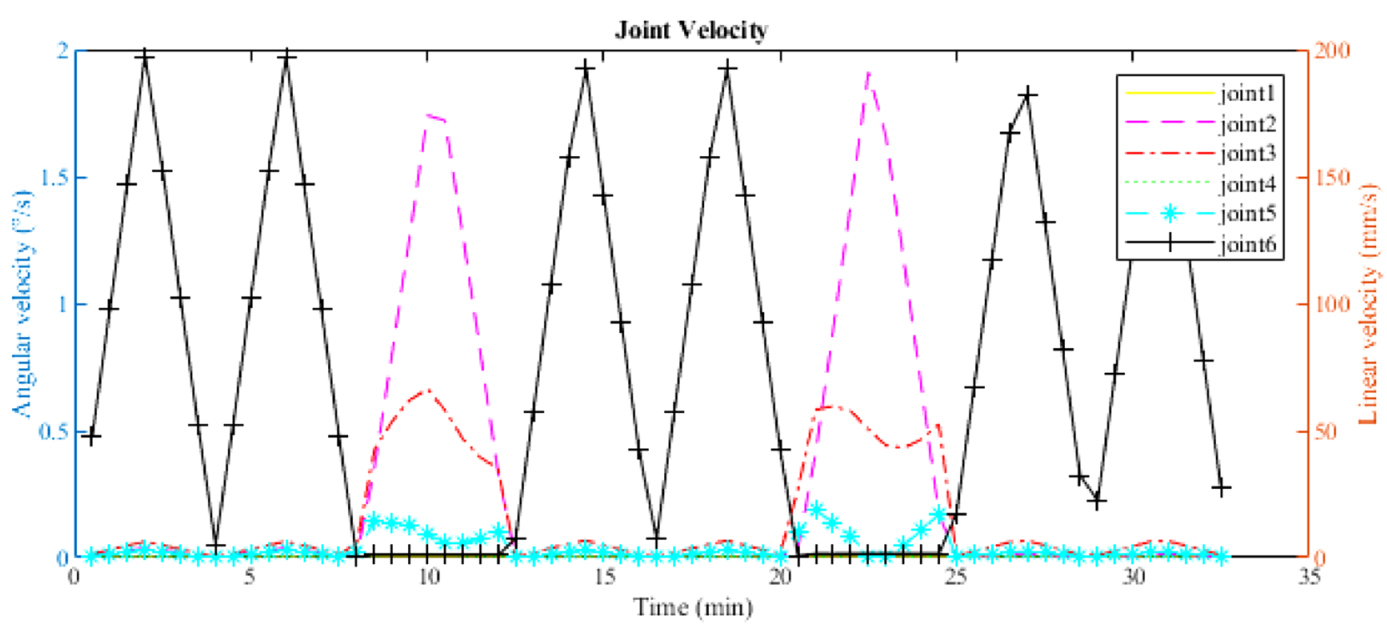

Figure. 9 Joint velocity

third, and fifth joints. Throughout the simulation process, the amount of rotation of joint 1 is very small, which avoids the impact of excessive energy consumption and end tremor due to the rapid rotation of joint 1 . At the same time, it has been verified that the maximum value of each joint speed is within the range allowed by the hydraulic system of the equipment. This operation planning method can be applied to the HPS3016C shotcrete manipulator.

\section{Shotcrete operation test and analysis}

In order to verify the correctness and operability of the above work, we conducted an automatic shotcrete test during the construction of Xiangshuwan No. 2 inclined shaft. The prototype of the automatic shotcrete test is the HPS3016C shotcrete manipulator. The control system of the shotcrete manipulator consists of upper computer, controller, sensing sample system and an interface communication system. Among them, the upper computer is responsible for completing the trajectory planning, sending the planned hydraulic cylinder piston position to the controller, and real-time receiving of the sampling data. The trajectory planning program is written in $\mathrm{VC}++$. Figure 10 shows the experimental test environment diagram.

The wall rock grade of Xiangshuwan No. 2 inclined shaft is grade III, the arch spacing is $1.1 \mathrm{~m}$, the single-step footage is $2.4 \mathrm{~m}$, the spraying volume is $20 \mathrm{~m}^{3} / \mathrm{h}$, and the delivery rate of accelerator is $500 \mathrm{Kg} / \mathrm{h}$. The experiment anticipates that the spray thickness is $1200 \mathrm{~mm}$, and finally the average thickness error is $27 \mathrm{~mm}$, and the maximum thickness error is $45 \mathrm{~mm}$. According to experience, the average thickness error of manual shotcreting operation is $30 \mathrm{~mm}$, therefore the operation accuracy of automatic spraying method has reached the level of manual operation. From the

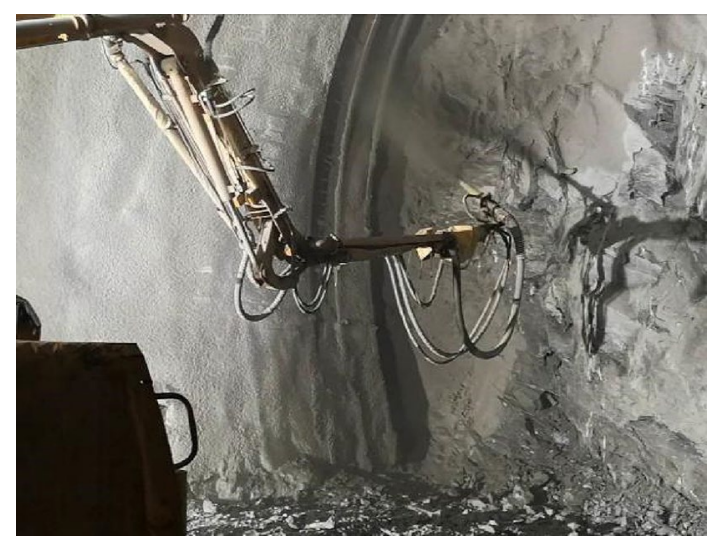

Figure. 10 Xiangshuwan No. 2 inclined shaft test's construction site

experimental results, it can be concluded that the designed system can realize the functions of full-automatic 3-d scanning, recognition of the sprayed surface, trajectory planning and motion control under the requirements of accuracy and has the ability of automatic spraying of the tunnel.

\section{Conclusion}

In order to improve the intelligent level of tunnel construction, this paper establishes a mathematical model of the shotcrete manipulator, designs an automatic spraying operation method of shotcrete manipulator and proposes an automatic spraying motion planning method based on block spraying for the spray surface model obtained by laser scanning, and uses this method to analyze the kinematics and path solving strategy of shotcrete manipulator. Finally, the effectiveness of this method is verified by simulation and experiment. This method can quickly and accurately analyze the kinematics 
of the shotcrete manipulator, and plan the trajectory of the manipulator. Later, this motion planning method will be widely applied to the full-servo shotcrete manipulator to realize the automatic spraying operation of the tunnel, which will greatly improve the quality and efficiency of the tunnel construction and provide technical support for the intelligent construction of the tunnel.

Acknowledgements This research was supported by the National Natural Science Foundation of China (Grant No. 51175143), China Postdoctoral Science Foundation (No. 2014M561338, No. 2017T100232) and Innovation project of Hunan Province (No. 2019GK1014).

Open Access This article is licensed under a Creative Commons Attribution 4.0 International License, which permits use, sharing, adaptation, distribution and reproduction in any medium or format, as long as you give appropriate credit to the original author(s) and the source, provide a link to the Creative Commons licence, and indicate if changes were made. The images or other third party material in this article are included in the article's Creative Commons licence, unless indicated otherwise in a credit line to the material. If material is not included in the article's Creative Commons licence and your intended use is not permitted by statutory regulation or exceeds the permitted use, you will need to obtain permission directly from the copyright holder. To view a copy of this licence, visit http://creativecommons.org/licenses/by/4.0/.

\section{References}

1. Vaibhav P, Nikhil T (2017) Construction of Tunnels, by New Austrian Tunneling Method (NATM) and by Tunnel Boring Machine (TBM). International Journal of Civil Engineering 6(6):25-36

2. Ono K (1996) Health and safety in shotcreting: ITA Working Group on Shotcrete Use in Tunnelling. Tunn Undergr Space Technol 11(4):391-409

3. L. Hu. Research and Application of Wet Shotcreting Machine in Underground Mine. Coal Science and Technology. 2009: 37 (07) :9-11+82.

4. Whitney DE (2007) Resolved Motion Rate Control of Manipulators and Human Prostheses. IEEE Transactions on Man-Machine Systems 10(2):47-53

5. Köker R, Çakar T, Sari Y (2014) A neural-network committee machine approach to the inverse kinematics problem solution of robotic manipulators. Engineering with Computers 30(4):641-649

6. Guo S, Cheng HJ, Xie CS (2013) An Algebraic Inverse Method of 7-DoF Manipulator Redundant Robots. Journal of Applied Sciences 13(13):2366-2371
7. Shimizu M, Kakuya H, Yoon W (2008) Analytical Inverse Kinematic Computation for 7-DOF Redundant Manipulators with Joint Limits and Its Application to Redundancy Resolution. IEEE Trans Rob 24(5):1131-1142

8. Zhang Q (2019) Unmanned rolling compaction system for rockfill materials. Automation in Construction 100:103-117

9. Ko S, Nakazawa A, Kurose Y (2017) Intelligent control of neurosurgical robot MM-3 using dynamic motion scaling. Neurosurg Focus 42(5):E5

10. Rowe WB, Chen Y, Moruzzi JL, Mills B (1997) A generic intelligent control system for grinding. Computer Integrated Manufacturing Systems 10(3):231-241

11. Cai ZX (2005) Advance in research of intelligent control and mobile robots. Journal of Central South University of Technology (Natural Science) 05:7-12

12. Girmscheid G, Moser S (2001) Fully Automated Shotcrete Robot for Rock Support. Computer-Aided Civil and Infrastructure Engineering 16(3):200-215

13. M.Y. Cheng, Y Liang. and C.M. Wey. Technological enhancement and creation of a computer-aided construction system for the shotcreting robot. Automation in construction, 2001: 10 (4) :517-526.

14. Berenstein R, Edan Y (2018) Automatic adjustable spraying device for site-specific agricultural application. IEEE Trans Autom Sci Eng 15(2):641-650

15. Cao ZY, Wang H, Wu WR, Xie JH (2013) Time-jerk optimal trajectory planning of shotcrete manipulators. Journal of Central South University (Science and Technology) 44(01):114-121

16. Tan GZ, Wang YC (2003) Theoretical and experimental research on time-optimal trajectory planning and control of industrial robots. Control Theory \& applications 20(2):185-192

17. Saramago SFP, Junior VS (2000) Optimal trajectory planning of robot manipulators in the presence of moving obstacles. Mech Mach Theory 35(8):1079-1094

18. Lai P, Samson C (2016) Applications of mesh parameterization and deformation for unwrapping 3D images of rock tunnels. Tunn Undergr Space Technol 58:109-119

19. Chettibi T, Lehtihet HE, Haddad M (2004) Minimum cost trajectory planning for industrial robots. European Journal of Mechanics 23(4):703-715

20. Liu SH (2011) Research for the automatic injection control technology with the concrete spraying robot. Construction Machinery Technology \& Management 24(3):105-108

21. Coulaud J-B, Campion G (2009) Gap-singular systems: definition and consequences in trajectory planning. Int $\mathrm{J}$ Control 82(5):837-848 